\title{
COMMENT
}

\section{Soft robot design methodology for 'push-button' manufacturing}

Jamie Paik (ID

'Push-button' or fully automated manufacturing would enable the production of robots with zero intervention from human hands. Realizing this utopia requires a fundamental shift from a sequential (design-materials-manufacturing) to a concurrent design methodology.

\section{Classic robot design}

Robots are characterized by the combination of chains of transmission links and joints, which define the kinematics of the system and dictate its degrees of freedom (DoF). Each active DoF requires a corresponding actuator that induces dynamic motion to the system. The performance of the system is then optimized through motor control to improve energy efficiency, position and torque accuracy as well as system bandwidth. This sequential design strategy does not allow spontaneous changes to the design of the robot because it is counter-intuitive, complex and costly.

\section{A new design paradigm}

Close human-robot interactions require robots to adapt to environmental changes and autonomously perform unforeseen tasks. Robotic behaviour that demands specific shapes and a range of motions that go beyond the initial design of the robot cannot be achieved through optimization of trajectory and force vectors based on motor control. Therefore, the design of the mechanism must be revisited, reiterated and reconfigured.

Interactive robotic behaviour can be implemented by augmentation of softness in the robotic design to complement, reconfigure and adapt to contingent assignments. The softness relates to both the software and the hardware of the robot; the design process demands more than just a component-wise design consolidation. This design strategy is different than the one applied for conventional robots and machines, sequentially following design parameters that are individually standardized by models of kinematics and dynamics, structural mechanics, materials, off-the-shelf motors and sensors, and established manufacturing processes.

Different approaches are being explored to fully embed functionality in soft robots using a single design file: the incorporation of a variety of distributed sensors into one device through 3D printing; 3D printing of the system based on a single file for printing but with a post-processing step to assemble the discrete components ${ }^{1}$; and the use of multiple materials from a single file, assuming that the materials are mostly polymers or packed in a polymeric matrix. The chemical interactions between the layers and embedded components can be designed, but the physics of these interactions is extremely complex. Therefore, the design of soft material robots is often guided by intuition and bio-inspiration. However, this intuition-based design strategy fails for complex materials and embedded discrete components.

Multimaterial and multicomponent-based robot manufacturing requires all design parameters to be concurrently considered, but there is currently no standard model available for simultaneous design and development. Origami robot or robogami design follows this new design approach ${ }^{2}$. The functionality of a robogami depends on the material and folding mechanism. The mechanical performance of the robot varies with composition, geometry and fabrication process. Consequently, there is no single solution and the design necessitates iterative fabrication and characterization tests to achieve the desired functionality. This issue can be addressed by the use of topology optimization, in which both structure and material distributions are simultaneously defined. The systemic categorization of the essential mechanical design features, such as geometry, mechanisms, functional materials and fabrication process, enables us to use specific models for each design consideration and ensures applicability of the proposed methodology.

Finally, a clear systematic design process should be established to facilitate robogami construction. The design methodology of robogami, simultaneously taking geometry, mechanisms, materials, discrete components and the fabrication process into account, creates an analogy to conventional robot design with defined sub-features, such as shape, kinematics, joints and actuation. However, the ongoing development of new methods and techniques in actuation, sensing, materials and fabrication requires conventional robot design to constantly adapt its design methodologies. A concurrent design strategy, reinforced with quantification and standardization, can produce universal applications for any machine design. 


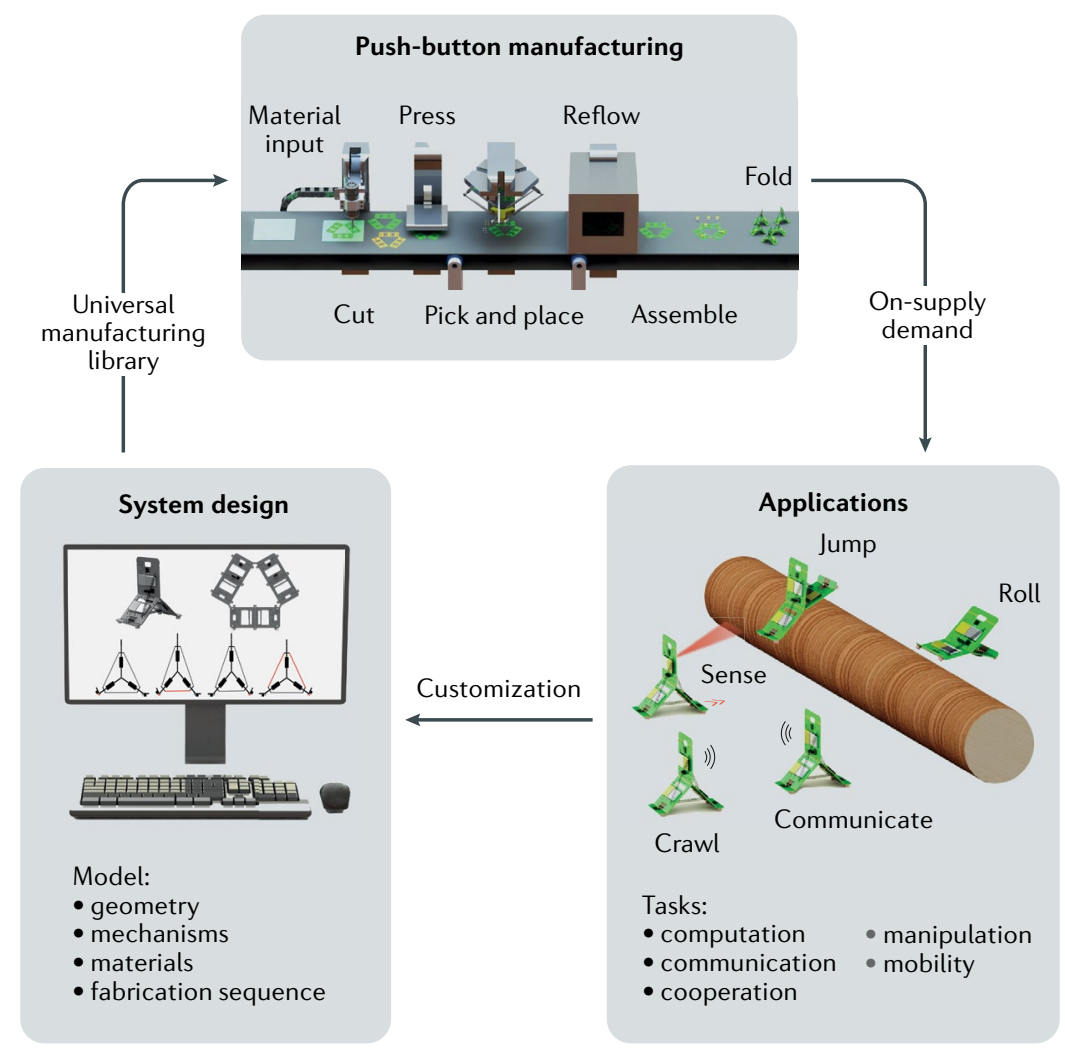

Fig. 1 | Push-button manufacturing. The universal manufacturing library determines the whole manufacturing process that assigns every fabrication process to make a robot for specific tasks. The concurrent design methodology uses a comprehensive model of the robot system to create the library. Adapted with permission from REF.', IEEE.

\section{A comprehensive model}

Applying this new design paradigm to robots requires not only an understanding of component-wise models regarding kinematics, mechatronics, materials and fluids, but also a comprehensive model of the overall system.

A comprehensive model can be applied to a design tool to define the system performance based on the actuator, geometric parameters and combination of materials. Design tools for soft pneumatic actuators (SPAs) use fundamental physics and principles of fluids to model simplified physical behaviours. For example, a software toolkit for soft robotics assumes a linear stressstrain relationship of soft material behaviours ${ }^{3}$. With this linear relationship assumption, this toolkit can simulate soft device deformation in real time. However, the nonlinear and viscoelastic nature of soft materials and the implementation of discrete components, such as embedded sensors, render the behaviour of the material more complex, making it challenging to predict the outcomes for applications that employ the highly deforming nature of the materials. Specific soft system designs, such as a heart ${ }^{4}$, pneu-net actuators and human vertebrae actuators, use a nonlinear finite element code to simulate different design parameters. Although computationally costly, finite element analysis can predict the stress-strain response of linear and bending actuators with highly nonlinear material behaviour. One of the materials used mostly for soft actuators is Ecoflex. The design tool can robustly simulate the mechanical performance, for example, displacement and blocked-force, of an Ecoflex-based SPA by characterizing its hyperelastic and viscoelastic behaviour with an appropriate material constitutive law ${ }^{5}$.

Control strategies for feedback processing are also important. The model-based strategy builds a model of a soft robot through experiments and then designs a controller to cancel out the errors between the output and the reference. The model-less strategy applies a deep learning-based control approach by building a relationship between the input and output force through deep learning of numerous real scenarios. A hybrid approach is implemented in current soft robots: a semi open-loop control with an empirical model-based bang-bang control. However, a close-loop control based on a comprehensive system-level model is necessary for reproducible and progressive research in soft robotics.

\section{Modern and organic manufacturing}

Push-button manufacturing enables the production of a fully functional robot from a single input command, without the need for post-assembly, without overstock or understock and waste, and with precise lead-time and cost estimation. The push-button manufacturing file contains not only the material and physical geometry parameters but also functionality, fabrication process and manufacturability of each component. Therefore, manufacturability is already embedded in the parameters used to generate the design. If the system cannot be fabricated or is not functional, the design file is not even made. The application and design methodology are part of the manufacturing loop, because the universal manufacturing library dictates the design parameters that are embedded with the manufacturing process for specific applications (FIG. 1).

3D printing of robots reflects this concept. For example, multimaterial printers (StrataSys, Markforged) are used to print actuator moulds or passive robotic structures $^{6}$ for low-profile origami robots. Smart materials react to external stimuli such as heat, UV light, humidity, magnetic or electrical fields and are explored for the printing of actuators, called $4 \mathrm{D}$ printing. $4 \mathrm{D}$ printing enables the fabrication of transferring patterns of different inks (water-, silver- and carbon-based) that undergo swelling and drying processes, thereby changing shape or resistance to achieve sequential folding through Joule heating or a focused laser beam ${ }^{7}$. Multimaterial printing can also be used to maintain the integrity of a structure or to endow the system with selective flexibility ${ }^{8}$. However, current printing technologies rely on the physical properties of polymers, often negatively affecting the performance of sensors and actuators. Design freedom in material choices is imperative for the development of more robust systems.

\section{Conclusion}

Printable robotics and soft robotics design methodologies are at the forefront of the push-button manufacturing approach, in which the design parameters include all information to verify and control the production. Therefore, the next generation of manufacturing files, for 
example, the universal manufacturing library code, will need to be compatible with both the new and the established manufacturing methods to allow the full extent of design freedom. Transforming this design process for applications beyond soft robot design and manufacturing will accelerate the journey to a new era of robotics, in which components and materials can be mixed and matched for a variety of applications.

1. Valentine, A. D. et al. Hybrid 3D printing of soft electronics. Adv. Mater. https://doi.org/10.1002/adma.201703817 (2017).

2. Zhakypov, Z. et al. Design methodology for constructing multimaterial origami robots and machines. IEEE Transactions on Robotics 34, 1-15 (2018).

3. Coevoet, E. et al. Software toolkit for modeling, simulation, and control of soft robots. Adv. Robot. 31, 1208-1224 (2017)

4. Roche, E. T. et al. A bioinspired soft actuated material. Adv. Mater. 26, 1200-1206 (2014).
5. Agarwal, G. et al. Stretchable materials for robust soft actuators towards assistive wearable devices. Sci. Rep. 6, 34224 (2016).

6. Zhakypov, Z. et al. The design and control of the multi-modal locomotion origami robot, Tribot. 2015 IEEE/RSJ International Conference on Intelligent Robots and Systems (IROS) https://doi.org/10.1109/ IROS.2015.7353994 (2015).

7. Shigemune, $\mathrm{H}$. et al. Origami robot: a self-folding paper robot with an electrothermal actuator created by printing. IEEE/ASME Transactions on Mechatronics 21, 2746-2754 (2016).

8. Naficy, S. et al. 4D printing of reversible shape morphing hydrogel structures. Macromol. Mater. Eng. 302, 1600212 (2017).

\section{Acknowledgements}

This work was supported by the Swiss National Science Foundation Funds (Robust: 205321_163292 and START: 200021_165694) and the Swiss National Centre of Competence in Research Robotics.

\section{Competing interests}

The authors declare no competing interests. 